\title{
Abdominal pseudohernia due to herpes zoster
}
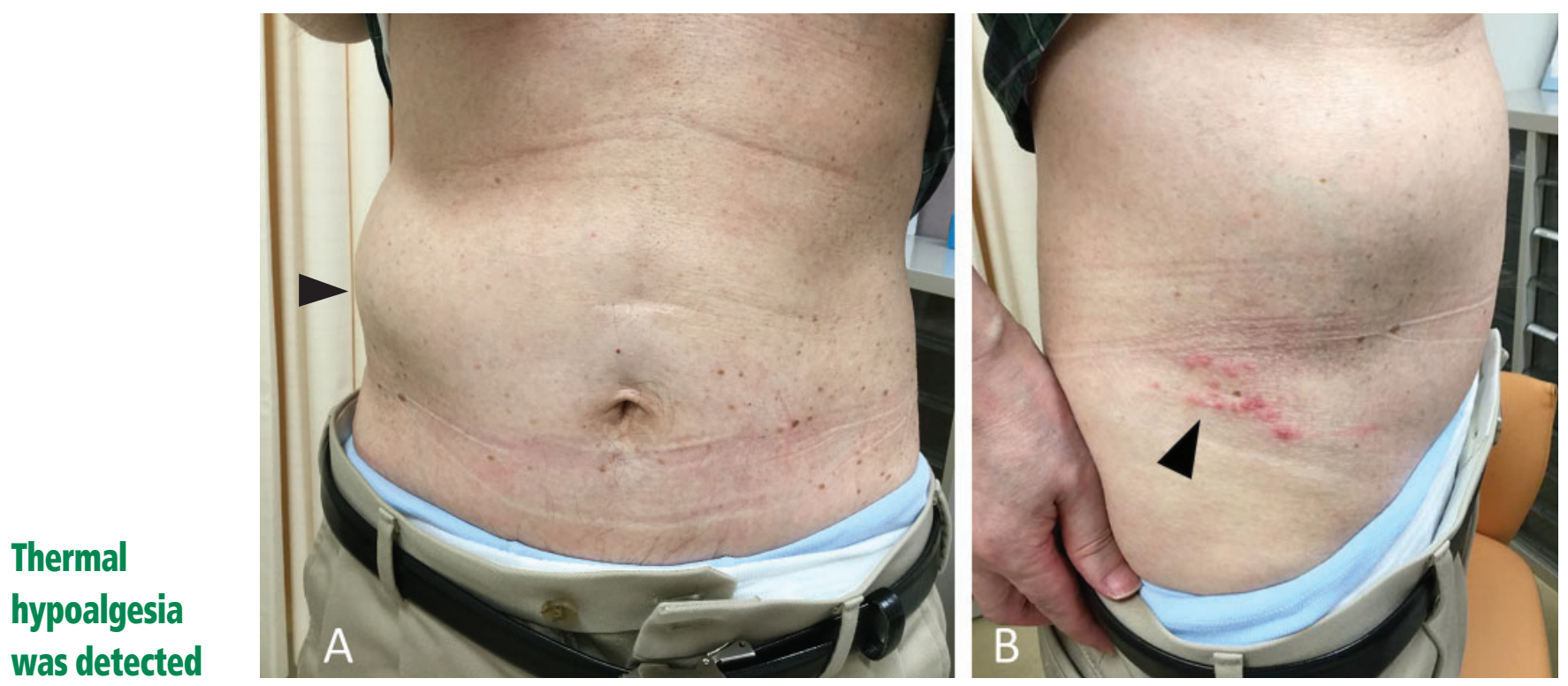

in a belt

from the lower

right side

of the abdomen to the back
Figure 1. (A) Bulging of the right abdominal wall (arrowhead). (B) The papules aggregated in a row on the right side of the lower abdomen (arrowhead).

A

66-yeAr-Old immunocompetent man $A$ presented with bulging and persistent burning pain in the right abdominal side for 4 days. Two days before the presentation, he went to another hospital and underwent abdominal computed tomography, which revealed no intraperitoneal abnormality.

On examination, the bulge was soft and nontender and did not have any palpable mass (Figure 1A). Thermal hypoalgesia was detected by alcohol swab in a belt from the lower right side of the abdomen to the back, a neurologic finding suggestive of zoster infection. No other neurologic deficit was seen. However, there were papules aggregated above the right groin (Figure 1B). The patient had doi:10.3949/ccjm.88a.20178 been unaware of this rash until it was pointed out. The rash was thus presumed to have developed on the morning of the presentation day because the patient confirmed that there was no skin change when taking a bath the previous night.

The diagnosis of abdominal pseudohernia due to herpes zoster was made based on the physical findings.

Lesions of herpes zoster often progress through stages, in the course of 7 to 10 days, from red macules and papules (as in this patient) to vesicles, to pustules and crusts, ${ }^{1}$ and then eventually subside spontaneously. ${ }^{2}$ Our patient was reassured that the condition would resolve on its own. He was prescribed valacyclovir 3,000 mg/day for 7 days. The bulging gradually improved over several weeks. 


\section{NEUROLOGIC COMPLICATIONS OF INFECTION}

Although skin neuralgia is a well-known complication of herpes zoster, the varicella-zoster virus can also cause motor neuron deficits, probably because of direct spread into spinal anterior horn cells and ventral roots. ${ }^{3}$

When the virus affects the lower thoracic spine, unilateral abdominal bulging may occur because of paresis of abdominal muscle. ${ }^{4}$ Most cases subside spontaneously, ${ }^{2}$ and patients can be reassured and thus be spared unnecessary diagnostic tests or medications.

Causes of pseudohernia other than herpes include diabetic radiculoneuropathy; trauma, such as ventral root injury from tumor removal and prolapsed intravertebral disc; and infection, such as Lyme disease and poliomyelitis. ${ }^{5,6}$

\section{KEYS TO THE DIAGNOSIS}

There are several keys to the diagnosis of abdominal pseudohernia due to herpes zoster.

First, the physician should not rule it out simply because there is no rash. Although the condition is often termed postherpetic abdominal pseudohernia, ${ }^{3,4}$ the bulging precedes the herpetic rash in nearly $10 \%$ of patients. ${ }^{2}$ This is why I prefer the term abdominal pseudohernia due to herpes zoster.

Second, a targeted history is important, eg, to rule out tick exposure and Borrelia-related neurologic complications. ${ }^{\text {? }}$

Third, imaging tests including magnetic resonance imaging may be indicated to check for mechanical compression of thoracic nerve roots if close follow-up does not reveal delayed-onset herpetic rash.

The combination of unilateral bulging of the abdominal wall and herpetic rash indicates abdominal pseudohernia due to herpes zoster. The rash may develop after the bulging, and thus, close follow-up may be of benefit.

Acknowledgments: We thank Robert Blakytny, DPhil, from Edanz Group for editing a draft of this manuscript.

\section{DISCLOSURES}

The author reports no relevant financial relationships which, in the context of his contribution, could be perceived as a potential conflict of interest.

\section{REFERENCES}

1. Sampathkumar P, Drage LA, Martin DP. Herpes zoster (shingles) and postherpetic neuralgia. Mayo Clin Proc 2009; 84(3):274-280. doi:10.1016/S0025-6196(11)61146-4

2. Chernev I, Dado D. Segmental zoster abdominal paresis (zoster pseudohernia): a review of the literature. PM R 2013; 5(9):786-790. doi:10.1016/j.pmrj.2013.05.013

3. Hung CT, Wang WM. Postherpetic pseudohernia. CMAJ 2012; 184(3):E202. doi:10.1503/cmaj.111481

4. Yagi Y, Matono T, Nakamura K, Imura H. Postherpetic abdominal pseudohernia: a diagnostic pitfall. J Gen Fam Med 2017; 19(1):3637. doi:10.1002/jgf2.147

5. Yoo J, Koo T, Park E, Jo M, Kim MS, Jue MS. Abdominal pseudohernia caused by herpes zoster: 3 case reports and a review of the literature. JAAD Case Rep 2019; 5(8):729-732. doi:10.1016/j.jdcr.2019.06.019

6. Oliveira PD, dos Santos Filho PV, de Menezes Ettinger JE, Oliveira IC. Abdominal wall postherpetic pseudohernia. Hernia 2006; 10(4):364-366; doi: 10.1007/s10029-006-0102-6.

7. Mormont E, Esselinckx W, De Ronde T, Hanson P, Deltombe T, Laloux P. Abdominal wall weakness and lumboabdominal pain revealing neuroborreliosis: a report of three cases. Clin Rheumatol. 2001;20(6):447-50. doi: $10.1007 / \mathrm{s} 100670170015$

Address: Junki Mizumoto, MD, Department of Medical Education Studies, International Research Center for Medical Education, Graduate School of Medicine, The University of Tokyo, 7-3-1 Hongou, Bunkyo-ku, Tokyo, 113-0033 Japan; jnk_mizu@yahoo.co.jp 